\title{
A Simplified Fractal Texture Analysis Approach using Quadtree Decomposition with Huffman Coding Technique
}

Rev. Sr. J. Rani ${ }^{1 *} \&$ Dr. G. Glorindal ${ }^{2}$

$I^{*}$ Research scholar, St. Eugene University, Zambia, Lilongwe, Malawi. Email: ranij79@ gmail.com

${ }^{2}$ DMI-St.John the Baptist University, Lilongwe, Malawi. Email: glorygj@yahoo.com, DOI: 10.46431/MEJAST.2021.4301

Copyright: (C2021 J.Rani \& G.Glorindal. This is an open access article distributed under the terms of the Creative Commons Attribution License, which permits unrestricted use, distribution, and reproduction in any medium, provided the original author and source are credited.

\section{ABSTRACT}

Fractal compression is compression of the lossy type, which is applied for natural textured images. A fractal image compression technique based on the Quadtree algorithm and Huffman coding is proposed in this work. Agonizingly, the term "fractal compression" refers to an image compression technique that uses the fractal geometry of the image data stream to achieve lossy compression. Realistic images and textures are created with the help of this tool. It is based on the fact that parts of an image are frequently similar to other parts of the same image, which allows for faster processing. The most widely used partitioning mechanism is image partitioning in a tree structure. In this emerging world of image processing quadtree partitioning is a one-of-a-kind technique that divides an image into a set of homogeneous regions. Huffman coding is a type of data compression that is lossless. The Huffman encoding algorithm is introduced through this technique, which creates an alphabetic list of all of the alphabet symbols, which is then arranged in descending order of their likelihood of occurring. The peak signal-to-noise ratio is quite improved by employing the proposed technique, and the encoding time is reduced.

Keywords: Image processing, Compression, Quadtree, Huffman coding.

\section{Introduction}

Compression technique has created an increasing important to enhance image storage and transmission. Due to redundancy and similarity across distinct parts in an image, compression is achievable. In these types of situations, fractal compression of images is a highly effective form of compression. Fractals are geometric or rough shapes that can be divided into portions, each of which is a smaller version of the whole due to a property known as self-similarity. Fractals can be divided into portions because they are self-similar to one another [1].

A mathematical fractal is based on an equation that is iterated repeatedly and is a type of recursive feedback. A common occurrence in nature is fractals. Across a wide but finite scale range, these artifacts have a self-similar structure. Nature's fractals, such as trees and ferns, may be computer-modeled using a recursive process [18]. In the early 1990s, Jaquin proposed using block forming as a basis for a fractal approach, and this idea has since been widely adopted. When it comes to compressing images, coder usage of square chunks known as "range" is widely employed. A domain block is four times as large as a range block in size. Once the image has been divided into smaller square blocks, those blocks are given the range blocks [2]. The search engine looks for the domain blocks and their rotations that have the best matches for each range block. Every range block is saved, along with the domain and any other relevant information to obtain that range [16]. As a result, rather than storing the entire range, only the parameters are retained, which results in compression.

The decoder reconstructs the original image through iterative procedures. Now because of the high compression ratio fractal compression is particularly helpful, the algorithm's decoding step is unaffected by the reconstructing image, and the reconstruction of an image is of high quality [17]. Using the difference between the two images, the peak signal-to-noise ratio (PSNR) is computed to determine the quality of the reconstructed image compared to the original image [7]. 


\subsection{Fractal Image Compression}

Fractal theory has placed a strong emphasis on indexing and compression techniques and the development of numerous post-processing methods applicable to this approach. While the advantages of fractal compression include rapid decompression, high bit rate, and resolution freedom, the primary disadvantage is the high processing cost associated with encoding and decoding. When compression is taken into consideration, fractal coding is unable to compete with other methods at this time.

On the other hand, almost all of the fractal coding applications currently in use are derived from image database indexing and face recognition applications, which are both commonplace. Some type of coding is employed, and this coding has a high degree of discrimination capability. Even though the PSNR for the coding module is very high, Given that fractal coding is frequently the technical stumbling block, it is preferable to make it as efficient as possible [3] As long as you keep the copy machine in a feedback loop, the final output will be determined by how well the input image is converted. For the final image to be infinite in size or dimension if the points in the copy were spread out, this technological limitation is reasonable. [4] Except for this requirement, the transformation can take on any shape. For choosing the shape transformations,

$$
w_{i}\left[\begin{array}{l}
x \\
y
\end{array}\right]=\left[\begin{array}{ll}
a_{i} & b_{i} \\
c_{i} & d_{i}
\end{array}\right]\left[\begin{array}{l}
x \\
y
\end{array}\right]+\left[\begin{array}{l}
e_{i} \\
f_{i}
\end{array}\right]
$$

An adequate amount is required in order to produce exciting plane transformations known as affine transformations. Input images can be stretched, rotated, and scaled with the help of these tools, which can also be translated. These transformations operate in a loopback mode, which means that the images produced are fractals. An affine transformation is a transformation that maps a plane to itself.

The affine transformation is represented as,

$$
w_{i}\left[\begin{array}{c}
x \\
y \\
z
\end{array}\right]=\left[\begin{array}{ccc}
a_{i} & b_{i} & 0 \\
c_{i} & d_{i} & 0 \\
0 & 0 & s_{i}
\end{array}\right]\left[\begin{array}{c}
x \\
y \\
z
\end{array}\right]+\left[\begin{array}{c}
e_{i} \\
f_{i} \\
o_{i}
\end{array}\right]
$$

The linear portion of the image is defined now by the variables a, b, c, and d, which control the image's rotation, skew, and scaling. Users can adjust the contrast and brightness of the image before and after transformation using the parameters $\mathrm{s}$ and $\mathrm{o}$.

\subsection{Contractive Transformations in Images}

Contractive transformations are defined as those that can be applied to any two points P1 and P2 [5]. The distance between P1 and P2 is calculated as,

$$
d\left(w\left(p_{1}\right), w\left(p_{2}\right)\right)<d s\left(p_{1}, p_{2}\right)
$$

Some have s that is less than 1 . As a result, applying a contractive map will always bring points closer together [5], as shown in the diagram. This concept applies to any space on which the function $d\left(\mathrm{P}_{1}, \mathrm{P}_{2}\right)$ can be defined, 
regardless of the type of space. If $\mathrm{P}_{1}=\left(\mathrm{x}_{1}, \mathrm{y}_{1}\right)$ and $\mathrm{P}_{2}=\left(\mathrm{x}_{2}, \mathrm{y}_{2}\right)$ are the coordinates of the points, then the following is true, then,

$$
D\left(p_{1}, p_{2}\right)=\sqrt{\left(\left(x_{2}-x_{1}\right)^{2}+\left(y_{2}-y_{1}\right)^{2}\right)}
$$

\subsection{Quadtree Decomposition}

Fractal Geometry has developed into a significant branch of modern mathematics and nonlinear science, with applications in a wide range of scientific and engineering fields. Two reviews on fractal compression encoding are currently being published; one examines the use of fractals in image compression, and the other examines the effect of fractals on sound compression [6]. Fractal encoding is inefficient because it takes an excessive amount of time to complete. It is possible to use fractal coding in conjunction with other coding methods to reduce encoding time while simultaneously improving image quality. Each square image is partitioned into four equal-sized square blocks, and each block is examined for homogeneity in the Quadtree image analysis method [18]. A block that does not meet the criteria for subdivision does not help to advance the investigation, and the criteria may need to be broadened to include such subdivisions. The procedure is repeated for each block until the specification is met, at which point the procedure is terminated.

\subsection{Huffman Encoding}

A list of all alphabet symbols in decreasing order of probability must be generated to begin using the Huffman encoding algorithm [8]. The binary tree is then constructed from the bottom up, with each leaf representing a unique symbol. This is accomplished in stages by selecting two symbols with the lowest probability of occurrence, relocating them to the top of the partial tree, deleting them from the list, and replacing them with an auxiliary symbol representing the two original symbols. When a tree can be reduced to a single auxiliary symbol or symbol, it is complete. Following that, the tree is traversed to determine the codewords associated with the symbols.

\subsection{Huffman Decoding}

Before beginning to compress a data file, the encoder must choose which codes will be used to compress the data. It follows the probabilities associated with the rates of occurrence of the symbols in question. It is necessary to include probabilities or frequencies in the output as side information for any Huffman decoder to decompress the data. Since the frequencies are all integers and the probabilities are all scaled integers, this is straightforward. The majority of the time, it only results in a slight increase in output size (a few hundred bytes).

However, the codes can be written directly to the output instead of being specified in a fixed-length format. This is inconvenient due to the variable-length formats. Writing the Huffman tree rather than the frequency values takes up significantly more space than simply printing the output. The decoder must locate the beginning of the compressed text, identify where it begins in the original text, and use this information to search for the corresponding Huffman tree, regardless of which strategy is employed.

Upon completing the remaining data, the system will automatically restart to read and translate any new information [9]. It is a straightforward decoding algorithm. Begin by reading the beginning of the input and starting 
at the first bit of the input (the compressed file). If the tree is empty, It should follow the edge; otherwise, It should follow the trunk. While continuing to read, move another edge closer to the leaves of the tree. Upon arriving at a leaf, the decoder receives the original, uncompressed signal, then converted it into a message. The method starts over from the beginning at the root $\mathrm{w}$, and the process starts over from the beginning at the root with the next bit, and so forth.

\section{Literature Review}

Image Compression Using Discrete Wavelet Transforms Based on Fractals IEEE Transactions on Image Processing published an article titled Using a Parallel Approach, written by Umesh B. Kodgule and B A. Sonkamble (2015) in which they describe their research [10]. The GPGPU technology from Nvidia is used in this article to apply a parallel approach to fractal image compression. To reduce the number of block comparisons, it is necessary to implement the discrete wavelet transform-based feature detection method described above. Two block classification and comparison methods, DWT and Parallel block classification and comparison, are being developed for use in fractal image compression to improve the encoder's performance [11].

Rasha Adel Ibrahim et al., proposed an improved fractal image compression technique incorporating quantized quadtrees and entropy coding. They published their findings in 2015 [12]. The compression of fractal images is improved by incorporating two previously used techniques, quantized quadtrees, and entropy coding, into the process. The quantization threshold value is used to divide the original grey-scale image into multiple blocks, which are then divided again. Entropy coding is used to improve the overall quality of the compression by increasing the amount of data that is compressed [13]. Their algorithm was evaluated in comparison to previous algorithms, and they discovered that the compression method they proposed could reduce encoding time by half. Aside from that, both the image quality and compression ratio have been improved.

Ming-Sheng Wu proposed a new method for fractal image compression that makes use of a genetic algorithm (GA) and discrete wavelet transformation, among other techniques (DWT) [14]. It has been demonstrated through experimentation and simulation that the proposed GA technique uses fewer MSE computations while simultaneously reducing the PSNR by 0.29 to $0.47 \mathrm{~dB}$. To make matters worse, the GA technique proposed in this study has an encoding time that is 100 times faster than the previous one, with acceptable trade-offs in terms of image quality recovery in 2013.

A DCT transformation is applied to the image before being compressed with fractal image compression to reduce the number of redundant compression operations performed on the image. Huffman coding is required for the encoding and decoding of images in both directions [15]. It will be possible to determine the effectiveness of the proposed approach to compressing color images once the system has been put into operation.

Fractal image compression is used to compress the image after it has been subjected to DCT. This helps to reduce the number of times the DCT compression procedure is applied to similar-sized blocks of the image, which reduces the amount of time the procedure takes. When encoding and decoding an image, it is necessary to use Huffman coding techniques. Upon completion of the implementation, the results will be analyzed to determine whether the proposed approach for compressing color images is effective. 
Table 1. Inference from the Previous Research Works

\begin{tabular}{|c|l|l|l|}
\hline S. No. & \multicolumn{1}{|c|}{ Name of Author } & \multicolumn{1}{|c|}{ Technique Used } & \multicolumn{1}{|c|}{ Result } \\
\hline 1 & $\begin{array}{l}\text { Umesh B. Kodgule } \\
\text { and B A. Sonkamble } \\
{[2015]}\end{array}$ & $\begin{array}{l}\text { Discrete Wavelet } \\
\text { Transformation using } \\
\text { parallel approach }\end{array}$ & $\begin{array}{l}\text { They recovered that visual effect and } \\
\text { average speed up ratio is good as } \\
\text { compare to other methods. }\end{array}$ \\
\hline 2 & $\begin{array}{l}\text { Rasha Adel Ibrahim } \\
\text { et.al. [2015] }\end{array}$ & $\begin{array}{l}\text { Quadtree and entropy } \\
\text { coding }\end{array}$ & $\begin{array}{l}\text { Proposed algorithm decreases encoding } \\
\text { time. }\end{array}$ \\
\hline 3 & $\begin{array}{l}\text { Ming-Sheng Wu } \\
{[2014]}\end{array}$ & $\begin{array}{l}\text { Genetic algorithm using } \\
\text { Discrete Wavelet } \\
\text { Transform }\end{array}$ & $\begin{array}{l}\text { The proposed GA method is 100 times } \\
\text { faster than the full search method. } \\
\text { PSNR value is reduced in comparison } \\
\text { with SGA method. }\end{array}$ \\
\hline 4 & $\begin{array}{l}\text { Rawat and Sukadev } \\
\text { Meher [2013] }\end{array}$ & $\begin{array}{l}\text { Discrete Cosine } \\
\text { Transformation }\end{array}$ & $\begin{array}{l}\text { Proposed method has high PSNR, } \\
\text { SSIM, UIQI value of compressed } \\
\text { images. }\end{array}$ \\
\hline
\end{tabular}

\section{The Proposed Algorithm}

The proposed algorithm partitions the original image using Quadtree decomposition. The dimensions range from 2 to 64 pixels. Following the completion of the Quadtree decomposition, the following information is stored in the programme: $\mathrm{x}$ and y coordinates, mean value and block size. It completes the Huffman coding of the image and calculates the compression ratio before proceeding to fractal coding. In the architecture shown in Fig.1., the image is reconstructed using Huffman decoding as in section 1.5, and the peak signal-to-noise ratio is calculated during the process. The method of fractal compression has been evaluated.

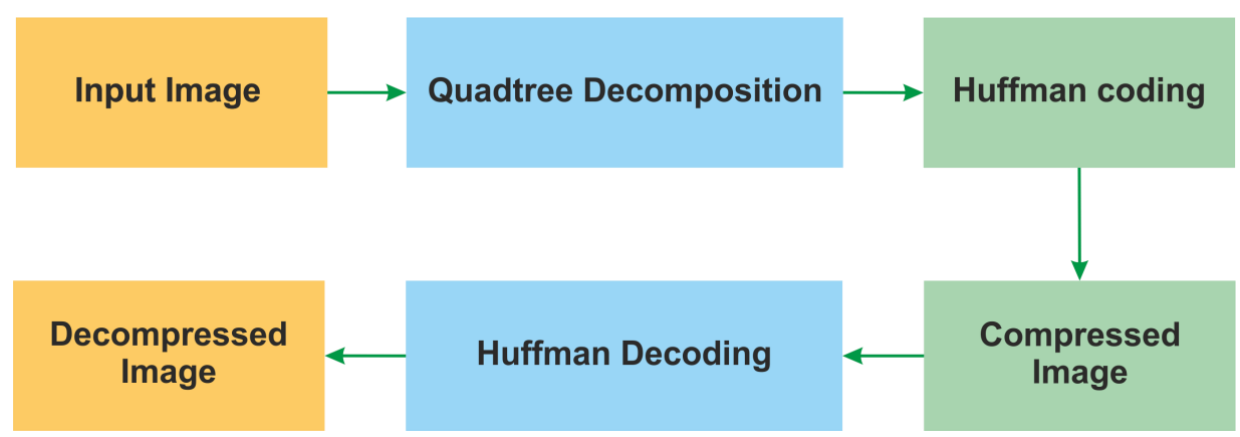

Fig.1. General Fractal Compression methodology

\section{Experimental Evaluation}

In addition to evaluating the algorithm on images, the algorithm was tested by using two similar but slightly different types of satellite images: an urban image with a resolution of 1243 pixels by 988 pixels, and a rural image with a resolution of 1045 pixels by 753 pixels, along with a 512-pixel by 512-pixel Lena image. Reducing the size 
of the images results in images that are 256 pixels wide and 256 pixels tall. Every value has a minimum and a maximum. Table 2 presents the compression ratio and PSNR of the Lena image, the urban satellite image, and the rural satellite image. The image from above is contrasted with the one below, demonstrating the differences that occur after Quadtree decomposition. While all three images have PSNR values above 31, the compression ratio varies from image to image in the results presented in Table 2. The city's PSNR (peak signal-to-noise ratio) is 32.9, which is greater than the national average, meaning that the city's visual compression ratio is 13.22 . When displayed in a rural environment using a compression rate of 10.12 and a PSNR of 31.29, the resulting images are of PSNR 31.29.

Compressed images while maintaining a PSNR of 34.29 are rendered at a PSNR of 34.29. As a result of structures, roads, and parks repeatedly appearing at fixed intervals, patterns are visible in the satellite image of an urban scene. Satellite images have a higher compression ratio because of fractals. Although urban features such as buildings, roads, and parks appear over and over in the built environment, resulting in patterns known as fractals, each of these features repeats itself infrequently.

In Quadtree fractal compression, this property decomposition/compression technique is made significantly easier. Even in rural areas, fractals are typically hidden because everything in rural areas is naturally occurring, such as plants, bodies of water, and dry soil. The technical limitations associated with reducing the size of rural imagery can quickly limit how quickly rural imagery can be compressed, limiting how quickly rural imagery can be compressed relative to urban imagery. This demonstrates that to attain zero fractals, the compression ratio of Lena's images yields zero.

Table 2. The Compression Ratios and PSNR Values

Derived for Satellite Imageries

\begin{tabular}{|c|c|c|c|c|}
\hline Test Images & $\begin{array}{c}\text { Time taken for } \\
\text { compression in } \\
\text { second }\end{array}$ & $\begin{array}{c}\text { Compression } \\
\text { Ratio }\end{array}$ & $\begin{array}{c}\text { Time taken for } \\
\text { decompression } \\
\text { second }\end{array}$ & $\begin{array}{c}\text { Peak Signal to } \\
\text { Noise Ratio in } \\
\text { dB }\end{array}$ \\
\hline Lena Image & 0.82 & 7.44 & 0.55 & 34.33 \\
\hline $\begin{array}{c}\text { Satellite Urban } \\
\text { Image }\end{array}$ & 0.39 & 13.22 & 0.45 & 32.9 \\
\hline $\begin{array}{c}\text { Satellite Rural } \\
\text { Image }\end{array}$ & 0.33 & 10.12 & 0.40 & 31.29 \\
\hline
\end{tabular}

When comparing three types of satellite images with relatively good PSNR values, the satellite images show compression ratios significantly more significant than the compression ratios found in the Lena image, as shown in the table above. It has been discovered through comparative analysis that the compression ratio of satellite imaging peak signal to noise ratios is meager and that the satellite has shallow peak signal to noise ratio values for both urban and rural satellite imaging. Following the analysis presented here, it is clear that the most efficient way to 
achieve the highest compression ratio is to combine fractal compression with quadtree decomposition. This approach is more appropriate for high-resolution urban satellite images than the other approaches discussed here.

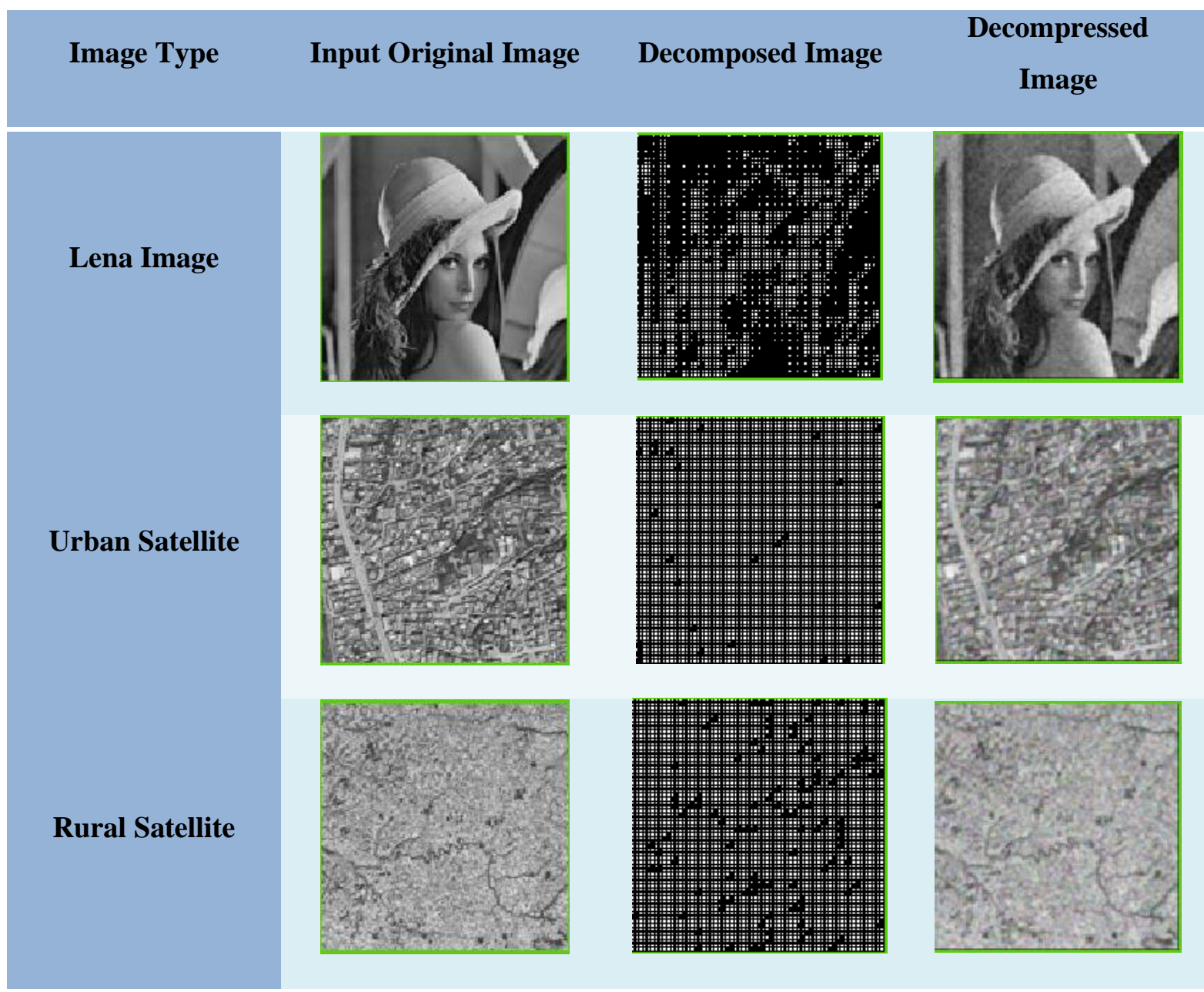

Fig.2. Image Conversion results

Following fractal compression, the images in the illustrations have been recreated. First part shows three images with considerably higher PSNR values than the original. Thus, the image quality has improved in the reconstructed image shown in Fig.2.

In cases where PSNR values greater than 31 are recorded, fractal compression with quadtree decomposition works well in creating high compression rates. In urban and rural areas, obtaining PSNR values above 90 is difficult.

\section{Conclusion}

On satellite images, it is possible to achieve compression ratios and PSNR values that are superior to the sum of the two algorithms working independently. When taken from space, satellite urban images are compressed at an average ratio of 13.22 with a peak signal to noise ratio of 32.9 , while rural satellite images are compressed at an average ratio of 10.12 with a PSNR 31.29. Compared to satellite rural and urban images, Lena images have the lowest compression ratio (7.44) and the highest PSNR (34.33) of any of the satellite systems. Thus, a simplified approach of Fractal compression has been implemented to attain a basic knowledge of image analysis and identification. 


\section{Declarations}

\section{Source of Funding}

This research did not receive any grant from funding agencies in the public, commercial, or not-for-profit sectors.

\section{Competing Interests Statement}

The authors declare no competing financial, professional and personal interests.

\section{Ethical Approval}

Not Applicable.

\section{Consent for publication}

Authors declare that they consented for the publication of this research work.

\section{Availability of data and material}

Authors are willing to share data and material according to the relevant needs.

\section{References}

[1] Chuang, Y. J., \& Wu, J. L. (2003, December). An SGH-tree based efficient huffman decoding. In Fourth International Conference on Information, Communications and Signal Processing, 2003 and the Fourth Pacific Rim Conference on Multimedia. Proceedings of the 2003 Joint (Vol. 3, pp. 1483-1487). IEEE.

[2] Distasi, R., Nappi, M., \& Riccio, D. (2005). A range/domain approximation error-based approach for fractal image compression. IEEE Transactions on Image Processing, 15(1), 89-97.

[3] Kekre, H. B., Sarode, T. K., Lahoti, A., Sawant, G., \& Sange, S. R. (2011). Image Reconstruction using Fast Inverse Half tone and Huffman Coding Technique. International Journal of Computer Applications, 975, 8887.

[4] Chen, J., Samuel, R. D. J., \& Poovendran, P. (2021). LSTM with bio inspired algorithm for action recognition in sports videos. Image and Vision Computing, 112, 104214.

[5] Zhao, J., Li, K., Xi, X., Wang, S., Saravanan, V., \& Samuel, R. D. J. (2020). Analysis of complex cognitive task and pattern recognition using distributed patterns of EEG signals with cognitive functions. Neural Computing and Applications, 1-10.

[6] Samuel, D. J., \& Cuzzolin, F. (2021). Unsupervised anomaly detection for a Smart Autonomous Robotic Assistant Surgeon (SARAS) using a deep residual autoencoder. arXiv preprint arXiv:2104.11008.

[7] Rajesh, R. S., Tamilselvia, G., \& Sivananainthaperumala, P. (2016). A New PAPR reduction Technique based on Precoding and Gamma Companding Technique for OFDM system. Scientific J. of PPI-UKM, 3(4), 182-186.

[8] Yu, H., Li, L., Liu, D., Zhai, H., \& Dong, X. (2010, November). Based on quadtree fractal image compression improved algorithm for research. In 2010 International Conference on E-Product E-Service and E-Entertainment (pp. 1-3). IEEE. 
[9] Mangalraj, P., Sivakumar, V., Karthick, S., Haribaabu, V., Ramraj, S., \& Samuel, D. J. (2020). A Review of Multi-resolution Analysis (MRA) and Multi-geometric Analysis (MGA) Tools Used in the Fusion of Remote Sensing Images. Circuits, Systems, and Signal Processing, 39(6), 3145-3172.

[10] Kodgule, U. B., \& Sonkamble, B. A. (2015). Discrete wavelet transform based fractal image compression using parallel approach. International Journal of Computer Applications, 122(16).

[11] Sengan, S., Arokia Jesu Prabhu, L., Ramachandran, V., Priya, V., Ravi, L., \& Subramaniyaswamy, V. (2020). Images super-resolution by optimal deep AlexNet architecture for medical application: a novel DOCALN. Journal of Intelligent \& Fuzzy Systems, (Preprint), 1-14.

[12] Ibrahim, R. A., Youssef, S. M., \& Elkaffas, S. M. (2015, November). An enhanced fractal image compression integrating quantized quadtrees and entropy coding. In 2015 11th International Conference on Innovations in Information Technology (IIT) (pp. 190-195). IEEE.

[13] Jayachandran, A., Andrews, J., \& Prabhu, L. A. J. (2019, October). Modified Region Growing for MRI Brain Image Classification System Using Deep Learning Convolutional Neural Networks. In International Conference on Innovative Data Communication Technologies and Application (pp. 710-717). Springer, Cham.

[14] Wu, M. S. (2014). Genetic algorithm based on discrete wavelet transformation for fractal image compression. Journal of Visual Communication and Image Representation, 25(8), 1835-1841.

[15] Selvi, S. A., Kumar, T. A., \& Rajesh, R. S. (2021). CCNN: A Deep Learning Approach for an Acute Neurocutaneous Syndrome via Cloud-Based MRI Images. In Handbook of Deep Learning in Biomedical Engineering and Health Informatics (pp. 83-102). Apple Academic Press.

[16] Thiruvikraman, P., Kumar, T. A., Rajmohan, R., \& Pavithra, M. (2021). A Survey on Haze Removal Techniques in Satellite Images. Irish Interdisciplinary Journal of Science \& Research (IIJSR), 5(2), 01-06.

[17] Rajmohan, R., Pavithra, M., Kumar, T. A., \& Manjubala, P. (2021). Exploration of Deep RNN Architectures: LSTM and Gru in Medical Diagnostics of Cardiovascular and Neuro Diseases. In Handbook of Deep Learning in Biomedical Engineering and Health Informatics (pp. 167-202). Apple Academic Press.

[18] Kumar, T. A., John, A., \& Kumar, C. R. (2020). 2. IoT technology and applications. In Internet of Things (pp. 43-62). De Gruyter. 